\title{
THE BINARY DIGITS OF A POWER
}

\author{
KENNETH B. STOLARSKY
}

\begin{abstract}
Let $B(m)$ denote the number of ones in the binary expansion of the integer $m>1$ and let $r_{h}(m)=B\left(m^{h}\right) / B(m)$ where $h$ is a positive integer. The maximal order of magnitude of $r_{h}(m)$ is $c(h)(\log m)^{(h-1) / h}$ where $c(h)>0$ depends only on $h$. That this is best possible follows from the Bose-Chowla theorem. The minimal order of magnitude of $r_{2}(m)$ is at most $c(\log \log m)^{2} / \log m$ where $c>0$ is an absolute constant.
\end{abstract}

1. Introduction. It seems difficult to say anything about the distribution of ones in the binary expansion of a surd such as $\sqrt{2}$ (but see P. Erdös [6, p. 215, Theorem 2] and J. Samborski [11]). We study the easier yet somehow related problem of digital distribution in powers of integers. Let $B(m)$ denote the number of ones in the binary expansion of $m$. It is known that $B(m)$ and $h^{-1} B\left(m^{h}\right)$, for a fixed integer $h$, have the same average order of magnitude, namely $(\log m) /(2 \log 2)$. In fact, stronger results for base 10 are proved in $\mathrm{H}$. Davenport and P. Erdös [3]; see also A. Besicovitch [1]. Here we investigate the pointwise relationship of these quantities.

Throughout, logarithms are to the base 2 unless otherwise specified.

DeFINITION. Let

$$
r_{h}(m)=B\left(m^{h}\right) / B(m) .
$$

THEOREM 1. Let $m$ and $h$ be positive integers greater than 1 . Then

$$
r_{h}(m) \leqslant 2(h \log m)^{1-1 / h},
$$

and this is best possible in that there is a constant $c(h)>0$, depending only on $h$, such that

$$
r_{h}(m)>c(h)(\log m)^{1-1 / h}
$$

infinitely often.

THEOREM 2. Let $m$ be a positive integer greater than 1. Then (trivial estimate) we have

$$
r_{2}(m) \geqslant\{[\log m]+1\}^{-1} .
$$

On the other hand, there are infinitely many integers $m$ such that

$$
r_{2}(m) \leqslant 4(\log \log m)^{2} / \log m .
$$

Received by the editors January 31, 1978.

AMS (MOS) subject classifications (1970). Primary 10A35, 10A40, $10 \mathrm{~L} 99$. 
It is well known that

$$
B(a b) \leqslant B(a) B(b)
$$

for positive integers $a$ and $b$, and examples such as

$$
(111)(100100)=(111111)
$$

show it is best possible. However, (1.5) with $a=b=m$ is usually far weaker than $(1.2)$ with $h=2$, since $(\log m)^{1 / 2}$ is usually much less than $B(m)$. For very precise statements of the value distribution of $B(m)$ see I. Kátai and J. Mogyoródi [8].

An extensive bibliography for the function $B(n)$, together with a historical survey, is given in [12]; see also [13], [14].

2. Proof of Theorem 1. Let $x=B(m)$ and

$$
r_{h}(m)=B\left(m^{h}\right) / B(m) .
$$

Then by (1.5) and the trivial estimate

$$
B(m) \leqslant[\log m]+1
$$

where $[u]$ denotes the greatest integer in $u$, we have

$$
r_{h}(m) \leqslant x^{-1} \min \left\{h \log m+1, x^{h}\right\} .
$$

For $x^{h} \geqslant h \log m$ we have

$$
r_{h}(m) \leqslant(h \log m)^{1-1 / h}+1 .
$$

Otherwise

$$
r_{h}(m) \leqslant x^{h-1}<(h \log m)^{1-1 / h}
$$

and (1.2) follows.

Our proof of (1.3) uses a notable result of Bose and Chowla.

Theorem 3 (Bose-Chowla). Let $h \geqslant 2$ be an integer. Then there are infinitely many integers $M$ for which there exist integers $a_{1}, \ldots, a_{M+1}$ such that

$$
1 \leqslant a_{1}<a_{2}<\cdots<a_{M+1}=M^{h}
$$

while every sum of the form

$$
a_{j_{1}}+\cdots+a_{j_{h}}, \quad 1 \leqslant j_{1} \leqslant \cdots \leqslant j_{h} \leqslant M+1
$$

is distinct.

Proof. See either [2] or [7, pp. 81-83].

We shall refer to this property of the $a_{i}$ as the distinct sums property.

Let $k=[\log h !]+1$. Choose $M \geqslant 3(k+1)$ and $a_{1}, \ldots, a_{M+1}$ so that the conclusion of Theorem 3 holds. Let $C(i)$ be the class of all $a_{j}$ such that

$$
a_{j} \equiv i \bmod (k+1), \quad 0 \leqslant i \leqslant k .
$$

For some $i_{0}$, the corresponding $C\left(i_{0}\right)$ has at least

$$
[(M+1) /(k+1)]=N+1
$$

elements. Subtract $i_{0}$ from each element of $C\left(i_{0}\right)$, remove the smallest (which 
might be zero), and label the elements of the transformed set as $y_{1}, \ldots, y_{N}$ in increasing order. Thus

$$
1 \leqslant y_{1}<y_{2}<\cdots<y_{N} \leqslant M^{h} \leqslant\{(N+2)(k+1)\}^{h},
$$

and the $y_{i}$ evidently have the distinct sums property.

Define $m$ by

$$
m=\sum_{i=1}^{N} 2^{y_{i}}
$$

Then

$$
m^{h}=\sum^{\prime} C\left(h ; h_{1}, \ldots, h_{N}\right) 2^{y_{1} h_{1}+\cdots+y_{N} h_{N}}
$$

where the $C\left(h ; h_{1}, \ldots, h_{N}\right)$ are the usual multinomial coefficients, bounded above by $2^{k}$, and the summation is over all

$$
\left(\begin{array}{c}
N+h-1 \\
N-1
\end{array}\right)
$$

vectors $\left(h_{1}, \ldots, h_{N}\right)$ satisfying

$$
h_{1}+\cdots+h_{N}=h .
$$

By writing the multinomial coefficients in binary, we obtain

$$
C\left(h ; h_{1}, \ldots, h_{N}\right)=\sum_{j=1}^{J} 2^{b\left(j ; h_{1}, \ldots, h_{N}\right)}
$$

where $J=J\left(h_{1}, \ldots, h_{N}\right)$, and

$$
0 \leqslant b\left(j ; h_{1}, \ldots, h_{N}\right) \leqslant k, \quad 1 \leqslant j \leqslant J .
$$

Upon combining (2.12) and (2.14) we obtain

$$
m^{h}=\sum^{\prime} \sum_{j} 2^{y_{1} h_{1}+\cdots+y_{N} h_{N}+b\left(j ; h_{1}, \ldots, h_{N}\right)} .
$$

To see that the exponents on the right of (2.16) are distinct, examine them modulo $k+1$. Since

$$
y_{i} \equiv 0 \bmod (k+1),
$$

the equality of two exponents implies that the corresponding $b(j ; \cdot)$ terms are congruent modulo $k+1$, and hence, by (2.15), are equal. But then the values of $\left(h_{1}, \ldots, h_{N}\right)$ must be the same for both exponents by the distinct sums property. Finally, (2.14) shows that the values of $j$ are identical, since the binary expansion of a number is unique. Hence

$$
N^{h-1} / h ! \leqslant N(N+1) \cdots(N+h-1) / h ! N \leqslant B\left(m^{h}\right) / B(m) .
$$

On the other hand, from (2.10) and (2.11), we have

$$
\log m \leqslant y_{N}+1 \leqslant\{(N+2)(k+1)\}^{h}+1 \leqslant 2^{h+1} N^{h}(k+1)^{h} .
$$

Upon raising both sides of (2.19) to the power $(h-1) / h$ and combining with (2.18), the result follows. 
3. Proof of Theorem 2. We begin by observing that

$$
B\left(2^{a}-2^{b}\right)=a-b, \quad a \geqslant b .
$$

From this it is easy to deduce that for

$$
A>a_{1}>a_{2}>\cdots>a_{q} \geqslant 0
$$

we have

$$
\begin{array}{r}
B\left(2^{A}-2^{a_{1}}-2^{a_{2}}-\cdots-2^{a_{q}}\right)=1+\left(A-a_{1}-1\right)+\left(a_{1}-a_{2}-1\right) \\
+\cdots+\left(a_{q-1}-a_{q}-1\right)=A+1-a_{q}-q .
\end{array}
$$

Now let $q \geqslant 1$, set $a(t)=2^{t}$ and

$$
S=S_{q}=\sum_{j=1}^{q} 2^{a(q+1)-a(j)+1}
$$

and define

$$
m=2^{a(q+1)}-S .
$$

Then from (3.2) we have

$$
B(m)=2^{q+1}+1-\left(2^{q+1}-2^{q}+1\right)-q=2^{q}-q .
$$

Now

$$
m^{2}=2^{2 a(q+1)}-2^{a(q+1)+1} S+S^{2} .
$$

The first $q-1$ squared terms from $S^{2}$ cancel out the first two terms on the right of (3.6), so

$$
m^{2}=2^{a(q+1)+2}+\sum_{i=1}^{Q} 2^{e_{i}}, \quad Q=q(q-1) / 2,
$$

where each $e_{i} \geqslant 0$ is an integer. Clearly

$$
B\left(m^{2}\right) \leqslant 1+[q(q-1) / 2]
$$

it is easily seen (though not needed here) that equality holds. Since

$$
2^{q} \leqslant \log m \leqslant 2^{q+1},
$$

the result follows.

By slightly perturbing the exponents in (3.3), we can create a wider (but still rather "thin") class of integers with small values of $r_{2}(m)$.

4. Remarks. It seems reasonable to expect that

$$
\lim \inf r_{h}(m)=0
$$

for every fixed $h \geqslant 2$, but I do not see how to prove this. Set

$$
R_{h}(T)=T^{-1} \sum_{m=1}^{T} r_{h}(m)
$$

I conjecture that $R_{h}(T) \rightarrow h^{\prime}$ as $T \rightarrow \infty$, where $1<h^{\prime} \leqslant h$, and that 


$$
R\left(2^{n}\right) \leqslant R\left(2^{n+1}\right)
$$

at least for all sufficiently large $n$.

\section{REFERENCES}

1. A. S. Besicovitch, The asymptotic distribution of the numerals in the decimal representation of the squares of the natural numbers, Math. Z. 39 (1934), 146-156.

2. R. C. Bose and S. Chowla, Theorems in the additive theory of numbers, Comm. Math. Helv. 37 (1962/63), 141-147.

3. H. Davenport and P. Erdös, Note on normal decimals, Canad. J. Math. 4 (1952), 58-63.

4. H. Delange, Sur la fonction sommatoire de la fonction "somme des chiffres", Enseignement Math. (2) 21 (1975), 31-47.

5. M. P. Drazin and J. S. Griffith, On the decimal representation of integers, Proc. Cambridge Philos. Soc. (4) 48 (1952), 555-565.

6. P. Erdö, On the irrationality of certain series, Nederl. Akad. Wetensch. Proc. Ser. A. 60 (1957), 212-219.

7. H. Halberstam and K. F. Roth, Sequences, vol. I, Clarendon Press, London, 1966.

8. I. Kátai and J. Mogyoródi, On the distribution of digits, Publ. Math. Debrecen 15 (1968), $57-68$.

9. D. J. Newman and M. Slater, Binary digit distribution over naturally defined sequences, Trans. Amer. Math. Soc. 213 (1975), 71-78.

10. I. Shiokawa and S. Uchiyama, On some properties of the dyadic Champernowne numbers, Acta Math. Acad. Sci. Hungar. 26 (1975), 9-27.

11. J. R. Samborski, Problem E2667, Amer. Math. Monthly 84 (1977), 567.

12. K. B. Stolarsky, Power and exponential sums of digital sums related to binomial coefficient parity, SIAM J. Appl. Math. 32 (1977), 717-730.

13. K. B. Stolarky and J. B. Muskat, The number of binary digits in multiples of $\boldsymbol{n}$ (in prep.).

14. K. B. Stolarsky, Integers whose multiples have anomalous digital frequencies, Acta Arith. (to appear).

Department of Mathematics, University of Illinois, Urbana, IllinOIS 61801 